\title{
Las oportunidades de la publicidad en un entorno globalizado: el mercado castellano leonés
}

\author{
David Alameda García | dalamedaga@upsa.es \\ Elena Fernández Blanco | efernandezbl@upsa.es \\ Irene Martín Martín I imartinma@upsa.es \\ Universidad Pontificia de Salamanca
}

Resumen: Frente al contexto de concentración y especialización que caracteriza al sector publicitario y de la comunicación estratégica nacional, se abre una realidad regional y local que dista en gran medida de éste en cuanto a los procesos, agentes, volumen de negocio, comercialización y gestión de los espacios publicitarios y actividades comunicativas desarrolladas. El presente artículo es fruto de una investigación subvencionada por la Junta de Castilla y León con el objetivo de profundizar en el conocimiento de la realidad de la industria publicitaria y de las relaciones públicas de la región, para desentrañar sus oportunidades de futuro.

Palabras clave: publicidad, relaciones públicas, industria, estructura.

Abstract: Opposite to the context of concentration and specialization that it characterizes to the advertising sector and of the strategic national communication, there is opened a regional and local reality that is far to a great extent from this one as for the processes, agents, volume of business, marketing and management of the advertising spaces and communicative developed activities. The present article is a result of a research subsidized by the Junta de Castilla y León to knowledge the reality of the advertising industry and of the public relations of the region, to discover his opportunities of future.

Key words: advertising, public relations, industry, structure. 


\section{Apuntes del contexto actual de la industria publicitaria}

La concepción más clásica de la actividad publicitaria y las relaciones públicas sufre serias modificaciones a partir de la década de los noventa, ya que en esta década se produce un importante punto de inflexión en la industria publicitaria y en la comunicación estratégica en general. A lo largo de los últimos años asistimos a un conjunto de cambios en la economía y aportaciones desde la tecnología que afectan no solamente a las estructuras y funcionamiento de la publicidad, sino que sobre todo descubren las dificultades y los propios límites del sistema publicitario en particular y del económico en general. Las transformaciones del entorno económico, la entrada de nuevos agentes al sistema publicitario, la aparición de nuevos medios de comunicación, la evolución de las necesidades de los anunciantes, la progresiva fragmentación de las audiencias y los cambios en los consumidores, la intensificación de la competencia, las transformaciones originadas por la irrupción de las nuevas tecnologías o la búsqueda de la especialización en el sector son algunos de los principales cambios que se dan cita en un entorno ahora global y que conllevan una redefinición del propio concepto de publicidad y sus prácticas.

Las empresas anunciantes han evolucionado durante los últimos años en tres direcciones: en la valoración de su propia identidad, en la instrumentalización de ésta dentro de la propia empresa y, por supuesto, en su relación con las agencias de publicidad y otros agentes del mercado. Ahora las condiciones de mercado son mucho más competitivas, lo que implica la puesta en práctica de técnicas de marketing progresivamente más sofisticadas y un pensamiento más global de la comunicación, en un entorno caracterizado por la gestión de la imagen corporativa como principio de diferenciación en el mercado y la búsqueda de un capital reputacional para las marcas y productos.

Desde el punto de vista de las empresas de publicidad y comunicación lo más destacable en este proceso de transformación son los continuos procesos de internacionalización de negocios y capitales, concentración publicitaria en macrogrupos de comunicación y la diversificación interna de sus actividades. WPP, Interpublic, Omnicom, Havas, Publicis y Aegis son los principales grupos publicitarios presentes en nuestro país, que en sus manos tienen la gran mayoría del negocio publicitario. A través de sus redes de agencias manejan las cuentas publicitarias de grandes anunciantes, pero además, prestan otro tipo de servicios con cientos de empresas especializadas en marketing, investigación, relaciones públicas, generación de contenidos o imagen de marca que completan la oferta de cara a los grandes inversores. Estos grupos son pocos en número, pero no en tamaño, ya que operan con decenas de redes y marcas para poder ofrecer un servicio acorde a las necesidades de sus clientes, ya sean grandes marcas que asignan sus cuentas a nivel mundial o pequeños y medianos anunciantes de los respectivos mercados locales. En contraste con lo anterior, en el mercado español siempre han existido pequeños grupos publicitarios que ofrecen servicios de publicidad, marketing, comunicación y relaciones públicas que se posicionan como alternativa a las grandes multinacionales. Grupos que con el tiempo se han profesionalizado 
y crecido, llegando en algunos casos a alcanzar un volumen y tamaño similar al de los grupos internacionales

En este contexto, las nuevas tecnologías e Internet han sido decisivas para el replanteamiento del escenario de la comunicación publicitaria, e incluso, el desbordamiento de las prácticas comunicacionales. Suponen un cambio en los hábitos de los consumidores respecto a la comunicación, la información, los sistemas de trabajo, el entretenimiento, los servicios y el mercado publicitario (con la multiplicación de soportes de comunicación, la fragmentación de las audiencias y el desarrollo de la interactividad).

En definitiva, la consolidación de la globalización como fenómeno social, económico, político, empresarial y cultural encuentra de modo directo su manifestación en la actividad publicitaria. A este fenómeno hay que unirle otros dos fenómenos relacionados entre sí: los cambios del modelo de consumidor/receptor de la comunicación y las transformaciones originadas por la irrupción de las nuevas tecnologías. Estas tres grandes fuerzas están transformando y afectando a la industria publicitaria tanto a nivel global como a nivel regional y local.

\section{El mercado local de la comunicación publicitaria}

Frente a este contexto de concentración y especialización que caracterizan fundamentalmente al panorama publicitario nacional, se abre una realidad regional y local que dista en gran medida de aquélla en cuanto a los procesos, agentes, volumen de negocio, comercialización y gestión de los espacios publicitarios y actividades comunicativas desarrolladas (ver figura 1). Así, nos enfrentamos a una estructura de la publicidad que presenta un menor grado de especialización y de rotación entre los diferentes agentes publicitarios. Las agencias periféricas se ven en la necesidad de ofrecer a sus clientes toda la gama de servicios comunicativos, funcionando como las clásicas agencias de servicios plenos o agencias generales, incluso en áreas de asesoramiento de marketing. Dado el escaso volumen de negocio de las agencias, se ven obligadas a ofrecer un servicio completo que, en muchas ocasiones, impide la especialización. Además, existe una clara falta de planificación de recursos en los mercados locales que se manifiesta en la falta de fuentes de información fidedignas sobre estudios de audiencias, difusión e inversión publicitaria en los medios locales, la presencia de tarifas "planas" que no llegan a reflejar la audiencia de los medios. 


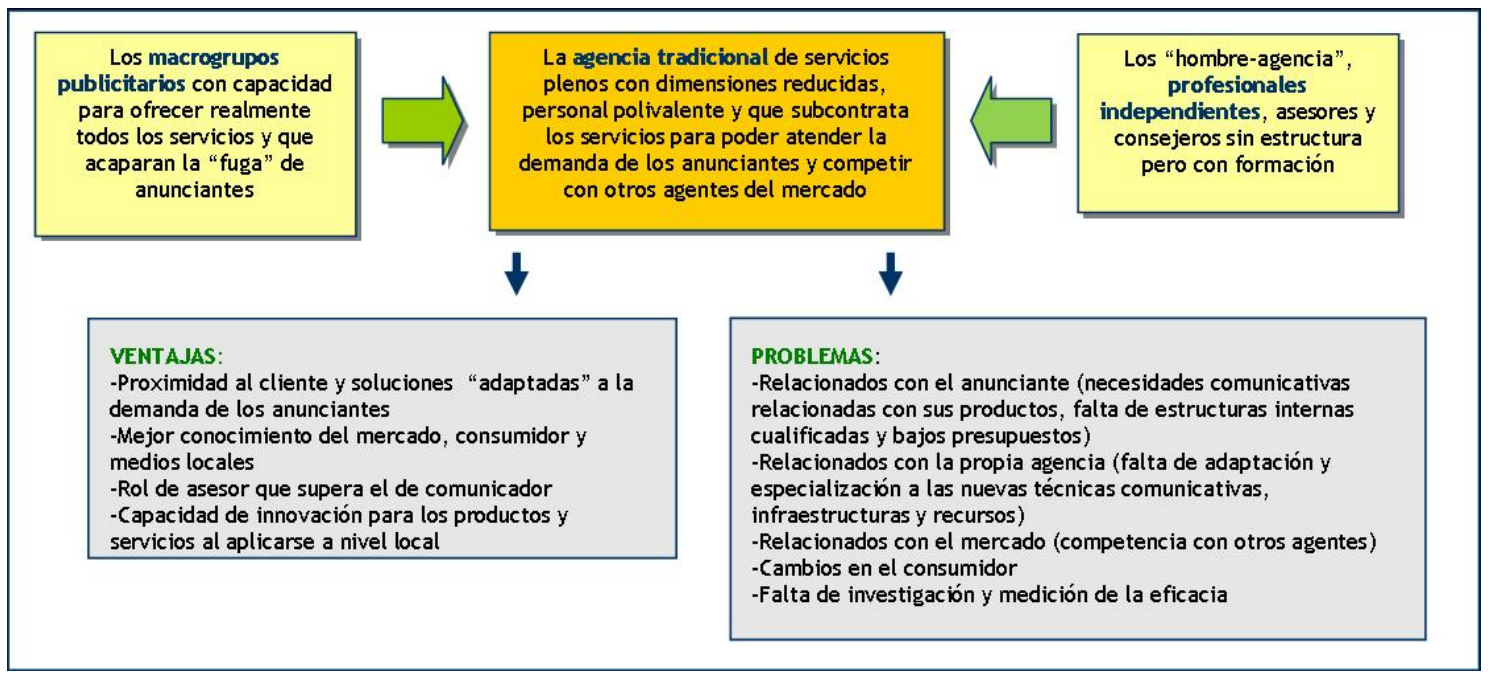

Figura 1.

Así resume Rafael López Lita el concepto de agencia local:

“La agencia local deberá ser de dimensión reducida o media, prestar sus servicios a clientes locales o a clientes globales exclusivamente en su ámbito local, los servicios predominantes se efectuarán en medios convencionales, desempeñando con frecuencia el rol de asesor; sus intangibles se vincularán fuertemente a la valía profesional de sus gestores; su dinámica de crecimiento será conservadora y su capital humano será versátil y con menor índice de rotación que en otro tipo de agencias" (2004:110).

Por otra parte, hay que destacar que en los mercados regionales y locales se presencia todavía una falta de cultura de comunicación tanto en los empresarios como en los trabajadores, dado que muchos de ellos se han incorporado a este mundo de la comunicación sin la formación adecuada ${ }^{1}$. Si bien hoy existen en los mercados regionales importantes facultades de comunicación con titulaciones específicas, todavía muchas de las empresas no exigen estas titulaciones para el desempeño de la labor comunicativa.

Observando diferentes investigaciones y publicaciones sobre el mercado local y regional de la publicidad definimos los principales problemas de la empresa de comunicación a nivel regional y local $^{2}$. Éstos podríamos agruparlos en dos direcciones:

\footnotetext{
${ }^{1}$ En este sentido encontramos numerosas voces en el sector que ponen de relieve esta situación y reclaman un aumento de la profesionalización. Véase VV.AA. "Agencias locales, medios locales" en LÓPEZ LITA, R., FDEZ. BELTRÁN, F. Y DURÁN MAÑES, A. (2004:111-124); ALMANSA MARTÍNEZ, A. "Publicidad y nuevas formas de publicidad local" en LÓPEZ LITA, R., FDEZ. BELTRÁN, F. Y DURÁN MAÑES, A. (2004: 129-136); MUT CAMACHO, M. "Internet o el futuro de la publicidad local" en LÓPEZ LITA, R... (2004: 349-355) entre otros.

2 Véase AGEP (2000): Empresas y agencias de publicidad. Estudio del mapa profesional del sector. Diagnóstico para un nuevo milenio. Asociación General de Empresas de Publicidad, Madrid; López Lita, R. (2004): "La agencia de publicidad local: hacia su conceptualización", en Questiones Publicitarias, no 9, vol. I, pp. 33-41; López Lita (2006): La comunicación corporativa en el ámbito local. V congreso de Comunicación Local. Castelló de la Plana: Publicaciones de la Universitat Jaume I; VISIEDO, R. (2000): "La oferta de publicidad de los 90 en la Comunidad Valenciana: el discurso de las agencias y los medios", en LAGUNA, A. (coord.): La comunicación en los 90. El mercado valenciano. Valencia, Servicio de Publicaciones Universidad Cardenal Herrera CEU.
} 
2.1. Problemas derivados del anunciante

Se trata de problemas que las agencias detectan provenientes del anunciante y que imposibilitan en algunas ocasiones el desarrollo de unos servicios publicitarios competitivos. Aquí se incluye la escasez de recursos de los anunciantes locales a la hora de invertir en publicidad (se trata de empresas medianas y pequeñas que para muchas la publicidad es considerada como un gasto, más que como una inversión y además, esta partida no da para todo y se centra, fundamentalmente, en obtener rápidamente resultados); poca estructura técnica y humana (estructuras comunicativas poco desarrolladas), necesidades comunicativas muy en la línea de la información de sus productos (lo que impide desarrollar estrategias de comunicación más centradas en los valores de las marcas); poca formación de los cuadros directivos y mandos intermedios en la función comunicativa de la propia empresa (falta de establecimiento de objetivos comunicativos lo suficientemente claros y precisos); y fuga de anunciantes (los anunciantes que cuentan con más recursos y demandan un servicio de mayor calidad y más infraestructuras de servicios auxiliares buscan agencias fuera del territorio donde operan, concretamente en el eje Madrid-Barcelona).

\subsection{Problemas derivados de la propia situación de cambio en el mercado}

Otra serie de problemas están relacionados con las tendencias generales del sector y que también tienen su presencia en el mercado regional y local. En este sentido se podrían considerar:

- La nueva realidad publicitaria está marcada por una gran competencia, un mercado muy fragmentado, en el que compiten por un cliente un elevado número de agencias. Esto está provocando, tanto a nivel nacional como local, una tendencia a la baja de la remuneración de los servicios publicitarios y un proceso natural de que sólo se mantienen aquellas agencias que logran adaptarse a las necesidades del mercado.

- Nuevas prácticas comerciales y pérdida de las funciones tradicionales de la agencia. En los últimos años el alto nivel de competencia se ha visto incrementado también por la aparición de nuevos agentes que se han especializado en funciones tradicionalmente desarrolladas por la agencia de publicidad. Es el caso de las agencias de medios, que han acaparado la función de medios. O el de las empresas de comunicación especializadas en diversos ámbitos: marketing directo, promociones, relaciones públicas, etc. que antaño, en mayor o menor medida, eran desempeñadas por la agencia tradicional de publicidad. Esta situación está generando cambios en los sistemas de retribución, guerra de precios, y que cada agente, por su parte, busque a sus clientes. 
- Cambios en las funciones de las agencias. Algunas agencias locales realizan ahora campañas cuya difusión no se circunscribe a su lugar de ubicación, sino que se extiende a todo el mercado donde concurren sus clientes. Igualmente, para no perder negocio, las agencias han tenido que realizar una extensión de línea, incorporando nuevos servicios de comunicación a medida que algunos anunciantes los han ido solicitando, pues si el cliente no los encuentra en su agencia, cambiará de proveedor. Es el ejemplo de Internet y el mercado online que las pequeñas agencias se ven obligadas a crear departamentos específicos o subcontratarlos para seguir siendo competitivos.

- La aparición de nuevas técnicas de comunicación y de nuevos medios como consecuencia de la saturación de los medios masivos conlleva a que los clientes busquen otras vías para la diferenciación de sus productos y marcas en el mercado. En este sentido, las agencias de publicidad (incluidas las locales) ven como principal problema cómo utilizar y desarrollar estrategias nuevas para captar la atención del consumidor.

- Los cambios en los hábitos del consumidor, en su relación con los medios, los nuevos accesos a la información y la posibilidad de interactividad están desbordando el sentido tradicional de la publicidad y plantean la necesidad de diseñar nuevas estrategias encaminadas a buscar el contacto y relación con el consumidor, así como incorporar nuevos métodos de medición de la eficacia comunicativa.

\section{Los retos de la publicidad en Castilla y León}

Para analizar la realidad local de la publicidad hemos tomado como caso de análisis la comunidad autónoma de Castilla y León. Para ello, la investigación realizada combina metodología de carácter cuantitativo y cualitativo. La investigación cuantitativa se centró en el análisis de las estructuras de la publicidad y las relaciones públicas en la región (agentes, estructuras, procesos de trabajo, funciones, sistemas de remuneración, etc.). Para ello, se seleccionaron el $50 \%$ del universo que componen las empresas de publicidad y rr.pp. en base a criterios de representación geográfica por ciudades y tamaño de las empresas. El trabajo de campo se realizó a través de cuestionario autoadministrado vía online. La fase cuantitativa se completó con otra de carácter cualitativo, que tenía como objetivo analizar los principales discursos, opiniones, expectativas y tendencias de los profesionales de la publicidad en la región. Se optó por entrevistas en profundidad a directivos/propietarios de algunas de las principales empresas de publicidad de Castilla y León (15 entrevistas).

En unas breves líneas ofrecemos unas pinceladas del mercado publicitario de Castilla y León, que nos permita comprender el discurso de sus profesionales y en definitiva los retos y oportunidades que se abren en el ámbito local. 
Castilla y León es una comunidad autónoma con un gran volumen de tejido empresarial, concretamente agrupa un 5,11\% de las empresas de España, que la sitúa como la sexta comunidad por importancia en número de empresas, tras Cataluña, Andalucía, Madrid, Comunidad Valenciana y Galicia. Sin embargo, esta relevancia no guarda relación con el escaso nivel de actividad publicitaria de los anunciantes castellano leoneses, apenas un 0,7\% de la inversión total nacional ${ }^{3}$. Hay por tanto una falta de correlación entre el entramado empresarial y las cifras de inversión, que deriva en una brecha importante entre la gestión de la publicidad "de las grandes cifras" y la publicidad periférica de los pequeños anunciantes.

En relación a las empresas de comunicación en la región nos encontramos ante un panorama rico, con más de 600 agentes $^{4}$, y claramente dominado por agencias de publicidad. Se trata de empresas pequeñas (con una media de 5 empleados frente a la media nacional que es de 21 empleados), con profesionales jóvenes (entre 31 y 40 años) en los que tan solo la mitad son licenciados. Los centros neurálgicos de la publicidad en la región son Valladolid, León y Burgos, copando más de la mitad del mercado. La filosofía y misión de las agencias y empresas de comunicación investigadas se puede resumir en tres grandes características: creatividad, servicios completos al cliente y experiencia en el sector. Agencias que, conscientes de las necesidades de sus clientes, adaptan sus servicios y sus recursos humanos de modo multidisciplinar. Es decir, se observa una proximidad a los clientes con soluciones adaptadas a sus demandas, ejerciendo más un rol de asesores.

Presentan una amplia oferta de servicios a sus clientes, que abarcan desde la creatividad hasta la comunicación interna; sin embargo, el 100\% de las empresas encuestadas coinciden en ofertar creatividad, diseño de páginas web, diseño e identidad visual y producción gráfica de las campañas. Los servicios más demandados por los anunciantes de Castilla y León se concentran en torno al diseño gráfico, la producción gráfica, la creatividad y los catálogos, anuarios y guías.

Las agencias presentan una organización sencilla, en torno a pocos departamentos que aglutinan numerosas funciones, siendo el departamento creativo y el de cuentas los imprescindibles para más del $75 \%$ de las empresas, seguidos de dirección y administración, medios y gestión de eventos. Por su parte, la función de medios es gestionada directamente por más de la mitad de las agencias, no siendo relevante en el mercado regional la figura de la agencia de medios, en claro contraste con la realidad nacional en la que las agencias de medios gestionan más del $70 \%$ del presupuesto publicitario de los anunciantes.

\footnotetext{
${ }^{3}$ La actividad publicitaria de los anunciantes de Castilla y León alcanza una inversión de 127,63 millones de euros durante el año 2007 y 113,78 millones en el año 2008, según datos de Infoadex. Y es que no podemos obviar que la inversión publicitaria en España está fuertemente concentrada entre Madrid y Cataluña. En el 2006, la Comunidad de Madrid polariza el 14,1\% de los anunciantes y el 52,2\% de la inversión publicitaria total. Cataluña, la segunda gran sede de la publicidad española, alberga al $15,6 \%$ de los anunciantes y el $19,7 \%$ de la inversión publicitaria total. En el resto de España, se asientan el $69,9 \%$ de los anunciantes cuyo presupuesto publicitario representa tan sólo el $27,6 \%$ de la inversión total estimada.

${ }^{4}$ Según los datos de la Cámara de Comercio (CAMERDATA 2008).
} 
Una vez esbozada de modo sucinto la estructura de la publicidad en Castilla y León, nos adentramos en los discursos de la publicidad regional, que se vertebran en torno a cuatro discursos principales, muy relacionados entre sí:

\subsection{Discurso de lo local: las ventajas de "ser local"}

Aunque en el discurso más explícito de los entrevistados el eje local-global no parece constituir un tema decisivo para el debate y el futuro de la publicidad en la región, la polaridad local/regional frente a lo nacional (en un sentido global) configura su manera de entender la comunicación publicitaria y de las relaciones públicas, los servicios, los procesos de trabajo, las relaciones con otros agentes y, en definitiva, el modo de afrontar el presente y futuro de la profesión.

El discurso de lo local es muy positivo y lleno de ventajas para las propias empresas de comunicación. Lo local, lo periférico, entendido como próximo, marca y define su propio posicionamiento como empresas pequeñas o medianas que garantizan un mejor conocimiento del mercado, del consumidor, de los medios y del cliente. Define su identidad en torno al "servicio total", entendido como el ofrecimiento al cliente de aquellos servicios que demande para dar soluciones a sus problemas concretos de comunicación. Es importante aclarar que el concepto de servicios plenos se refiere a su grado de adaptación y polivalencia, y no a la existencia real de profesionales y departamentos especializados en los diferentes servicios de la comunicación.

La visión local afecta también al modo de entender la publicidad por parte de los anunciantes que, en términos generales, están alejados de una óptica estratégica y entienden la comunicación desde el corto y medio plazo, como una ayuda para el desarrollo de su negocio. Aquellos anunciantes que, por el contrario, adoptan una manera de entender la comunicación global y a largo plazo, deciden contratar a las grandes agencias nacionales/internacionales para sus campañas, mientras que las agencias locales quedan relegadas a las adaptaciones de dichas campañas en el ámbito local.

Desde la visión de lo local o periférico se sienten cómodos y, en cierta manera, protegidos de la realidad publicitaria de las tendencias, de las grandes cifras o de los servicios más profesionalizados. Esto explica la falta de diferenciación de las agencias y que otras, estén dando el salto a lo global desde lo local y reclamen la importancia de la planificación, la recuperación del concepto de campaña y el concepto de marca; características que definen el modo de entender la publicidad en estos momentos.

\subsection{Discurso de la falta de cultura de comunicación}

Se detecta una ausencia de cultura en torno a la comunicación, que afecta tanto a anunciantes, como a agencias como a los propios medios de comunicación y resto de 
agentes sectoriales. Este problema presenta múltiples manifestaciones entre las que podemos destacar:

- Falta de inversión. Hay una escasa confianza en la publicidad y/o falta de recursos. Para gran parte de los anunciantes la comunicación publicitaria constituye un gasto y no una inversión a medio y largo plazo.

- Percepción de escaso apoyo institucional. Falta de respaldo por parte de las instituciones al sector, observándose un profundo desencuentro entre las agencias y la administración pública. En el fondo de dicho discurso subyace una demanda de sentido de comunidad.

- Falta de corporativismo. El escaso volumen de actividad publicitaria se refleja también en la falta de iniciativas, actividades comunes y asociacionismo para impulsar socialmente la profesión.

- Falta de posicionamiento de las agencias de comunicación. Ante las demandas de los anunciantes regionales, más enfocadas al planteamiento comercial y corto plazo, las agencias no requieren un posicionamiento especializado en el mercado.

- Fuga de los grandes anunciantes. Aquellos anunciantes que han evolucionado en sus planteamientos hacia estrategias integradas y con un horizonte global de la comunicación, mantienen su actividad en la región, pero todo lo concerniente a la comunicación lo delegan en los grandes núcleos de la publicidad (Madrid y Barcelona).

- Intrusismo y falta de profesionales especializados.

\subsection{Discurso del conflicto}

El discurso del conflicto deriva de los dos anteriores, puesto que la peculiaridad de lo local y, sobre todo, la ausencia de cultura de comunicación en la región provocan situaciones de conflicto entre los diferentes agentes. Los puntos de fricción son constantes y siempre relacionados con la competencia en diferentes ámbitos, percibida como una amenaza.

- Conflicto con anunciantes. El conflicto se vertebra en torno a estos ejes: a) Los grandes anunciantes contratan a agencias nacionales/internacionales; y b) El tipo de anunciante local impide desarrollar campañas innovadoras. Este conflicto es mutuo, porque los anunciantes afirman que las agencias no les comprenden y no se adaptan a sus planteamientos comunicativos.

- Conflicto con medios. Centrado en la competencia que ejercen los medios a la hora de la compra y venta de los espacios publicitarios, e incluso de la producción publicitaria. 
- Conflicto con agencias. Surgen del reparto de la pequeña tarta publicitaria de la región y del surgimiento de agencias no cualificadas que compiten por los mismos clientes.

- Alejamiento con la universidad. Se manifiesta en la falta de iniciativas comunes e incluso el desconocimiento por parte de los profesionales de los planes de estudio y los conocimientos que se imparten en la universidad. Esto mismo ocurre en el sentido contrario, desde la universidad se desconoce la realidad del mercado regional y local.

\subsection{Discurso de la oportunidad: querer ser}

Existe una clara contradicción entre el "ser" y el "querer ser". Mientras que los profesionales de la región se muestran orgullosos de trabajar en lo local, en constante sintonía y cercanía con el cliente, en un mercado que permite un aprendizaje mucho más global (se aprende a hacer de todo) y en el que la calidad de vida es mucho mejor; a la vez, añoran un comportamiento más maduro de los anunciantes, con una cultura comunicativa superior y con una mayor consideración de la publicidad.

Este "querer ser" también se manifiesta cuando desde el sector profesional se desea mayor dinamismo, se confía en un mercado con posibilidades, se apuesta por las nuevas tecnologías, se pide apoyo institucional para el desarrollo del sector y mayor visibilidad social.

Este discurso lleva implícita muchas oportunidades para el impulso de la publicidad en la región. Es decir, se aprecia que la publicidad y las relaciones públicas están en un momento de crecimiento y desarrollo, y todo ello a pesar de la crisis económica. Este impulso ya comienza a vislumbrarse a través de iniciativas y planteamientos de desarrollo como:

- entender lo local como oportunidad, para que con una alta profesionalización se conciba la comunicación en un sentido global. Ofrecer una visión global a la solución de la comunicación, con un asesoramiento al cliente en $360 \%$.

- el nacimiento de agencias con visión estratégica,

- acciones conjuntas de visibilidad social (como los Premios de la Publicidad de Castilla y León),

- el impulso institucional,

- el nacimiento de un canal regional de TDT que abre infinitas posibilidades a la publicidad,

- el acercamiento progresivo del mundo profesional y universitario, 
- el propio crecimiento de las facultades de publicidad en la región.

\section{Conclusiones}

Las principales conclusiones del estudio ponen de manifiesto que Castilla y León, a pesar de ser una comunidad con un extraordinario potencial para el mercado publicitario, necesita mayor impulso y dinamización. El sector publicitario, con una actividad muy prolífica y un volumen destacable de empresas (agencias de publicidad, empresas de relaciones públicas, agencias de comunicación, estudios de diseño gráfico, identidad corporativa, etc.), trabaja para mejorar la comunicación de las pequeñas, medianas y grandes empresas e instituciones de la región y su actividad repercute en el quehacer diario y el desarrollo eficaz de las empresas de sectores económicos importantes para la región, como pueden ser el de agroalimentación, automoción, telecomunicaciones o las propias instituciones públicas, entre otros.

No obstante, según apuntábamos, el sector está marcado por una gran red de pequeñas agencias que carecen de un posicionamiento diferenciado en el mercado, en tanto que ofertan un servicio total, un "todo para todos". A la falta de posicionamiento se suman otros problemas claramente identificados por sus protagonistas: fuga de grandes anunciantes de la región, falta de cultura de comunicación de los anunciantes, ausencia de asociacionismo y corporativismo, intrusismo profesional, competencia desleal de los medios, entre los más importantes. Sin embargo, estos puntos de fricción conviven con otras características propias de la región, como son la cercanía, el conocimiento profundo del mercado y los consumidores y la adaptación a sus clientes.

Desde los planteamientos de los profesionales, tanto en la fase cuantitativa como en la cualitativa de investigación, parece dibujarse un territorio en el cual se puede plantear una concepción diferente de la actual, más centrado en aprovechar los puntos fuertes de la realidad nacional de las grandes agencias (pensamiento transversal, estrategias integradas, modelo participativo, vuelta al concepto de marca, etc.) para sumarlos a las fortalezas del ámbito local (cercanía, versatilidad, conocimiento del mercado, etc.), todo ello desde un grado máximo de profesionalización (ver figura 2). 


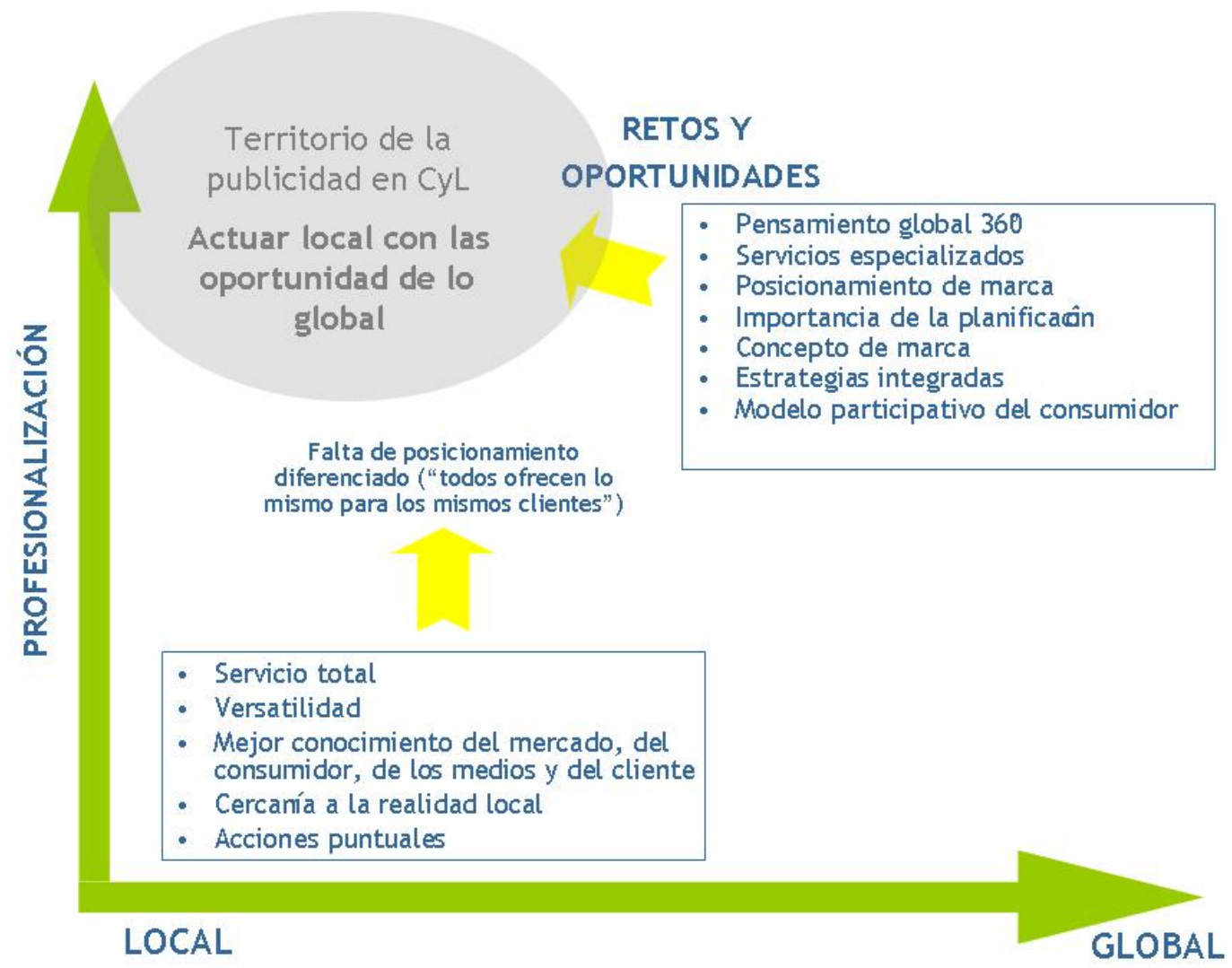

Figura 2.

Una vez sentadas las bases de esta línea de investigación de la comunicación estratégica en la región, con un primer diagnóstico del sector, se hace necesario un análisis del mapa profesional que permita conocer en profundidad los sistemas y procesos de la publicidad y las relaciones públicas en Castilla y León. Solo desde esta investigación, estaremos en condiciones de poder ofrecer algunas de las claves y tendencias que permitan definir un claro posicionamiento estratégico de los principales agentes.

\section{REFERENCI AS BI BLI OGRÁFI CAS}

AGEP (2000): Empresas y agencias de publicidad. Estudio del mapa profesional del sector. Diagnóstico para un nuevo milenio. Asociación General de Empresas de Publicidad, Madrid.

Alameda García, David, Fernández Blanco, Elena y Martín Martín, I rene (2008): La industria de la publicidad y de las relaciones públicas en Castilla y León (Proyecto de investigación: Junta de Castilla y León, Referencia PON03B07) Salamanca, Facultad de Comunicación, Universidad Pontificia de Salamanca. Alameda GarcíA, David (2007): Una nueva realidad publicitaria. La generación de valores corporativos en publicidad. Madrid, Laberinto. 
ARCEO VACAS, J.L. (coord.) (2004): Las relaciones públicas en España. Barcelona, Mc Graw Hill.

BARQUERO, J. D. (2005): Comunicación estratégica: relaciones públicas, publicidad y marketing. Barcelona, Mc Graw Hill.

CAstellblanque, M. (2006): Perfiles profesionales de publicidad y ámbitos afines. Barcelona, Editorial UOC.

CONSEJO SUPERIOR DE CÁMARAS DE COMERCIO (2008): "La empresa en España". Servicio de Estudios Cámaras de Comercio.

FNEP y AESAP (2002): Publicidad para el nuevo milenio. Primera reunión de profesionales de la publicidad en Salamanca. Salamanca, FNEP y AESAP.

GRUPO CONSULTORES (2008): Estudio de agencias de publicidad 2008. Madrid, Grupo Consultores.

INFOADEX (2008): Estudio Infoadex de la inversión publicitaria en España 2008. Madrid, Infoadex.

Medina, A. (2004): Apuntes para un cambio de siglo publicitario. Madrid, Cinca.

OCAÑA, F. (2006): La publicidad contada con sencillez. Madrid, MAEVA.

PABLOS, C. et al (2006): Dirección y gestión de los sistemas de información en la empresa. Madrid, ESIC.

LÓPEZ LITA, R., Fernández Beltrán, F. Y Durán Mañes, A. (eds.) (2004): La publicidad local. Castelló de la Plana: Publicaciones de la Universitat Jaume I.

LÓPEZ LITA, R. (2004): “La agencia de publicidad local: hacia su conceptualización”, en Questiones Publicitarias, no 9, vol. I, pp. 33-41.

LóPEZ LITA, R. (et. al.) (2006): La comunicación corporativa en el ámbito local. V congreso de Comunicación Local. Castelló de la Plana: Publicaciones de la Universitat J aume I.

VICTORIA, J. S. (ed.) (2005): Reestructuras del sistema publicitario. Barcelona, Ariel.

VISIEDO, R. (2000): “La oferta de publicidad de los 90 en la Comunidad Valenciana: el discurso de las agencias y los medios", en LAGUNA, A. (coord.): La comunicación en los 90. El mercado valenciano. Valencia, Servicio de Publicaciones Universidad Cardenal Herrera CEU.

[Recibido: 25-06-2009. Aceptado (con indicación de modificaciones): 04-12-2009. Recibido con modificaciones: 16-12-2009] 